\title{
Monocyte Activation Test (MAT) Reliably Detects Pyrogens in Parenteral Formulations of Human Serum Albumin
}

\author{
Rolando Perdomo-Morales ${ }^{1, *}$, Zenia Pardo-Ruiz ${ }^{1, *}$, Ingo Spreitzer $^{2}$, Alicia Lagarto ${ }^{1}$, \\ and Thomas Montag ${ }^{2}$ \\ ${ }^{1}$ Center for Pharmaceuticals Research and Development, Havana, Cuba; ${ }^{2}$ Paul Ehrlich Institute, Langen, Germany
}

\begin{abstract}
Summary
Disadvantages of the regulatory pyrogen test to assure safety of the end-product Human Serum Albumin (HSA) for parenteral use call for the implementation of an alternative test. In the current study, 16 HSA batches were assayed for pyrogens in parallel with the Rabbit Pyrogen Test, conventional and endotoxin-specific LAL assay and monocyte activation test (MAT).

It was found that all HSA batches were contaminated with $(1,3)-\beta$-glucans, which interfere with the conventional LAL. Endotoxin-specific LAL was not suitable to test HSA due to unacceptable endotoxin recovery. Experiments combining polymyxin $B$ and MAT demonstrated that pyrogenic batches were mainly contaminated with endotoxins. However, endotoxin-specific LAL failed to detect one of them. The contaminating (1,3)- $\beta$-glucans enhanced the MAT/IL-6 response to endotoxin, but not that of $M A T / I L-1 \beta$. The endotoxin equivalent concentrations obtained using the IL-6 readout were usually higher than those using $I L-1 \beta$, probably owing to the direct induction of IL-6 release from monocytes by (1,3)- $\beta$-glucans.

The MAT correlates with the rabbit pyrogen test, providing a higher safety level for pyrogenicity testing of HSA and probably other therapeutic proteins.
\end{abstract}

Keywords: pyrogen, albumin, (1,3)- $\beta$-glucans, endotoxin, monocyte activation test

\section{Introduction}

Pyrogens are fever-inducing substances usually derived from microorganisms that could induce deleterious responses to the host, including septic shock or even death, when entering the blood stream (Dinarello, 2004). Therefore, manufacturers of pharmaceutical parenteral products and medical devices that will be in contact with the systemic circulation are responsible for ensuring that their products are non-pyrogenic.

There are now three official pharmacopoeial tests to detect pyrogens in parenteral products: the Rabbit Pyrogen Test (RPT), the bacterial endotoxin test (BET), often referred to as the Limulus Amebocyte Lysate test (LAL), as well as the Monocyte Activation Test (MAT), of which there are five variants.

Due to the high consumption of animals required by RPT and its ethical implications, this test has been replaced by LAL in most pharmacopoeial monographs. However, most regulators continue to recommend the RPT for the evaluation of plasmaderived medicinal products.

The MAT is based on the in vitro activation of human monocytoid cells by pyrogens, which leads to the release of proin- flammatory cytokines that are determined by ELISA (Hartung et al., 2001). Five variants of the MAT have recently been standardized and validated (Hoffmann et al., 2005). They have all been accepted by the European Centre for the Validation of Alternative Methods (ECVAM) as alternatives to the RPT (ESAC Statement, 2006) and were adopted by the European Pharmacopoeia in 2010 (EP, 2010).

Nevertheless, according to the Interagency Coordinating Committee on the Validation of Alternative Methods (ICCVAM), the MAT cannot be considered a complete replacement of the RPT, due mainly to the lack of comparative data between the two methods. However, it can be employed as a substitute for detecting Gram-negative endotoxin in parenteral drugs on a case-by-case basis (ICCVAM, 2008, 2009). The same position has been adopted by the US Food and Drug Administration (FDA) (FDA, 2009).

Blood-derived product manufacturing presents specific challenges in relation to microbiological contamination, due to the possibility of initial contamination of plasma units and to the risk of introducing bacterial contaminations during the subsequent production steps. Moreover, as the main fever induc-

* Both authors contributed equally to this work.

Received March 14, 2011; accepted in revised form July 5, 2011. 
ing cytokines interleukin-1 $1 \beta$ (IL-1 $\beta$ ), interleukin-6 (IL-6), and tumor necrosis factor alpha $(\mathrm{TNF} \alpha)$ are natural constituents of human plasma, they can also be found in plasma pools for fractionation (EMEA, 2009). The European Medicines Agency (EMEA) encourages the replacement of RPT by alternative tests such as LAL or MAT in plasma-derived medicinal products (EMEA, 2009).

Human serum albumin (HSA) is an important plasma-derived medicinal product that has been used for more than 60 years as a parenteral therapeutic agent for the maintenance and restoration of blood volume (Mc Clelland et al., 1990). There are several reports on HSA products that have passed RPT and LAL but have elicited pyrogenic episodes in patients (Pool and McLeod, 1995; Poole et al., 1988a,b; Steere et al., 1978; Taktak et al., 1991), indicating that these tests do not always provide sufficient safety support.

Therefore, an improved safety testing strategy for pyrogens is required for HSA. Previous comparative studies between MAT and RPT on HSA showed that the whole blood MAT variant (WB-MAT) resulted in the same level of safety as the RPT (Spreitzer et al., 2002). Also, the MAT was more effective in detecting endotoxin at the borderline concentration, which often causes the RPT to fail, resulting in further assays with additional animals. Others have also found that MAT is a promising test system for pyrogenic contaminations of HSA (Poole et al., 1988a,b; Taktak et al., 1991).

Here we have carried out a parallel comparison of RPT, LAL, and MAT as end-product pyrogen test for HSA preparations. Samples of the 16 batches were not modified or artificially spiked with pyrogens, providing a real-life picture of the performance of each test. The MAT demonstrated the highest safety level for detection of pyrogens in HSA.

\section{Animals, materials, and methods}

\section{Samples and reagents}

In this study, sixteen batches of end-product 20\% HSA for parenteral use (referred to as batch $\mathrm{A}$ to $\mathrm{P}$ ) were assayed for pyrogens by the methods described below. The $2^{\text {nd }}$ International Standard for Endotoxin (NIBSC code: 94/580, UK) (Poole et al., 1997), reconstituted and stored as recommended in the manufacturer's instructions, was used as standard in MAT as described elsewhere (Andrade et al., 2003). The International Standard of human IL-1 $\beta$ (rDNA derived, NIBSC code: 86/552, UK) (Poole and Gaines-Das, 1991), and WHO $1^{\text {st }}$ International Standard of human IL-6 (NIBSC code: 89/548, UK) (Gaines-Das and Poole, 1993), were reconstituted in phosphate-buffered saline (PBS) containing 3\% bovine serum albumin (BSA) (Sigma, St. Louis, MO, USA) and stored at $-80^{\circ} \mathrm{C}$ until use. Dilutions of cytokine standards for ELISA were done in the same buffer. All consumables were purchased sterile and certified pyrogen-free. Glassware was depyrogenated by dry-heat at $250^{\circ} \mathrm{C}$ for $1 \mathrm{~h}$.

\section{Determination of endotoxin limit concentration (ELC)}

The endotoxin limit concentration was calculated as $E L C=K / M$, with $\mathrm{K}$ being the threshold pyrogenic dose of endotoxin per kilo- gram of body mass per day (120 EU/ $\mathrm{kg} / 24 \mathrm{~h})$ and $\mathrm{M}$ the daily maximum recommended bolus dose of product per $\mathrm{kg}$ body mass (FDA, 1987). Maximum dose of HSA according to the Cuban national formulary of pharmaceuticals is $6 \mathrm{~g} / \mathrm{kg} / 24 \mathrm{~h}$ (FNM, 2006), which is equivalent to $30 \mathrm{ml} / \mathrm{kg} / 24 \mathrm{~h}$ for $20 \%$ HSA. Therefore, an endotoxin limit of $4 \mathrm{EU} / \mathrm{ml}$ was used throughout this study as pass/fail criterion for LAL and MAT.

\section{Rabbit pyrogen test}

RPT in 20\% HSA was carried out according to the monograph described in the European Pharmacopoeia (EP, 2007) by injecting $5 \mathrm{ml}$ per $\mathrm{kg}$ of body weight.

\section{Limulus amebocyte lysate assay}

Determination of endotoxin by LAL was conducted by the kinetic chromogenic method using the Pyrochrome ${ }^{\circledR}$ kit (Associates of Cape Cod, E. Falmouth, MA, USA), according to the manufacturer's instructions. The reaction was kinetically measured at $405 \mathrm{~nm}$ and $37^{\circ} \mathrm{C}$ for one hour with a microplate reader Biotek Elx $808^{\mathrm{TM}}$ IU (BioTek ${ }^{\circledR}$ Instruments, Winooski, VT, USA). The data was processed with $\mathrm{KC}^{\mathrm{TM}}{ }^{\mathrm{M}}$ v.3.4 (BioTek ${ }^{\circledR} \mathrm{In}-$ struments, Winooski, VT, USA). The level of interference in the samples and its dilutions was assessed using endotoxin spikes at $0.5 \mathrm{EU} / \mathrm{ml}$. Where indicated, LAL reagent was reconstituted with Glucashield ${ }^{\circledR}$ buffer (Associates of Cape Cod), producing an endotoxin-specific LAL reagent (ES-LAL). HSA samples were diluted in borosilicate tubes (Associates of Cape Cod) to avoid endotoxin adsorption to polystyrene tubes promoted by albumin as described by Yokota et al. (2001). The maximum valid dilution (MVD) for HSA was 1:400.

\section{Monocyte activation test using fresh human whole blood (WB-MAT)}

a) Human whole blood incubation

Blood from healthy volunteers was drawn using syringes (Multifly ${ }^{\circledR}$, Sarstedt, Nümbrecht, Germany) connected to sodium heparin coated tubes (S-Monovette ${ }^{\circledR}$, Sarstedt, Nümbrecht, Germany). Human whole blood incubations were performed as described elsewhere (Daneshian et al., 2009; Spreitzer et al., 2002). In brief, $100 \mu 1$ of two-fold serial dilutions of HSA in saline were mixed with $1 \mathrm{ml}$ saline and $100 \mu 1$ fresh human blood in $1.5 \mathrm{ml}$ polypropylene reaction tubes (Eppendorf, Hamburg, Germany). The tubes were shaken gently by inversion and incubated overnight $(16-24 \mathrm{~h})$ at $37^{\circ} \mathrm{C}$. Thereafter, samples were shaken again and centrifuged for $5 \mathrm{~min}$ at $13,000 \mathrm{rpm}$. Supernatants were stored at $-80^{\circ} \mathrm{C}$ until cytokine measurements by ELISA were performed.

\section{b) Enzyme-linked immunosorbent assay (ELISA)}

Cytokine measurements were carried out with an in-house sandwich ELISA, based on matched antibodies against human IL-1 $\beta$ or IL-6 (Pierce Biotechnology, Rockford, IL, USA) diluted with PBS containing 3\% BSA. $50 \mu 1$ of $2 \mu \mathrm{g} / \mathrm{ml}$ coating monoclonal anti-IL- $1 \beta$ or $1 \mu \mathrm{g} / \mathrm{ml}$ coating monoclonal anti-IL-6 was added to each well of a microtiter plate (F96 Nunc-Immuno Plate, Polysorp, Nunc, Roskilde, Denmark) and incubated for $16-24 \mathrm{~h}$ at $4^{\circ} \mathrm{C}$. The plates were coated with $200 \mu 1$ blocking 
buffer (PBS containing 3\% BSA) for $2 \mathrm{~h}$ at $25^{\circ} \mathrm{C}$, and washed three times with PBS containing 0.5\% Tween 20 (Sigma, St. Louis, MO, USA). Supernatant $(50 \mu 1)$ from the whole blood incubation and $50 \mu 1$ of biotin-labeled monoclonal antibody $(0.25 \mu \mathrm{g} / \mathrm{ml}$ anti-IL- $1 \beta$ or $0.5 \mu \mathrm{g} / \mathrm{ml}$ anti-IL-6) were added per well and further incubated for $2 \mathrm{~h}$ at $25^{\circ} \mathrm{C}$. After washing four times, $100 \mu 1$ of streptavidin-peroxidase (Sigma, St. Louis, MO) were added into each well and incubated for $30 \mathrm{~min}$. Then plates were washed eight times and $100 \mu 1$ of 3,3',5,5'tetramethylbenzidine (TMB) (Sigma, St. Louis, MO, USA) was added. After 10-15 min the reaction was stopped with $50 \mu 11 \mathrm{M} \mathrm{H}_{2} \mathrm{SO}_{4}$. The absorbance was measured at $450 \mathrm{~nm}$ with $630 \mathrm{~nm}$ as reference wavelength in a microplate reader Biotek Elx $808^{\mathrm{TM}}$ IU (BioTek ${ }^{\circledR}$ Instruments, Winooski, VT). The MAT response to endotoxin was assessed from the dose-response plot of endotoxin standard concentration (from 0.03125 to $2 \mathrm{EU} / \mathrm{ml}$ ) vs. absorbance. As MAT can detect pyrogens other than endotoxins, results were presented as endotoxin equivalent units per $\mathrm{ml}$ (EEU/ml) as suggested by Montag et al. (2007).

c) Test for interferences

Demonstrating the absence of interfering factors is required to guarantee reliable results of the assay. The test was conducted by incubating diluted whole blood with sample dilutions spiked with $0.5 \mathrm{EU} / \mathrm{ml}$ and assayed in parallel with the corresponding unspiked dilution. Dilutions with endotoxin recovery within the $50-200 \%$ range were considered interference-free, and the first was defined as minimum valid dilution (MinVD).

d) Determination of IL-1 $\beta$ and IL-6 concentrations Where indicated, cytokine concentrations of undiluted HSA samples were determined by the ELISA procedure described above, along with the corresponding cytokine standard curve ranging from 6 to $500 \mathrm{pg} / \mathrm{ml}$ for IL- $1 \beta$ and from 1 to $200 \mathrm{pg} /$ $\mathrm{ml}$ for IL-6. Cytokine concentrations were obtained by linear regression of the linear portion of the curve of cytokine concentration vs. absorbance.

e) Differentiation between endotoxin and non-endotoxin pyrogen contamination

HSA samples that failed RPT were incubated with Polymyxin B sulphate (PMB) (Sigma, St. Louis, MO) to elucidate whether they were contaminated with endotoxin or not (Pool et al., 1999). The sample diluted in its MinVD was incubated with whole blood and $1 \mathrm{mg} / \mathrm{ml} \mathrm{PMB}$ (final concentration in the tube) (Huszar et al., 2002). Negative control treated with PMB was employed as assay control. IL- $1 \beta$ was measured by ELISA. Statistical significance was determined by using one-way ANOVA with GraphPad Prism 5.0 (GraphPad software, San Diego, CA).

\section{Results}

\section{Rabbit pyrogen test}

Batches G, H and I failed the RPT according to the European Pharmacopoeia guidelines (see the resumed comparative study in Tab. 3), i.e. these batches showed pyrogenic contamination.

\section{LAL assay}

It is well established that a valid LAL test requires the absence of interference (inhibition or enhancement) in the endotoxin activity, which is determined by the level of endotoxin recovery from positive product controls (spike) (FDA, 1987). The LAL method used in the current study requires an endotoxin recovery between 50-200\%. Typical results of the interference assay using conventional LAL in 10 batches are depicted in Table 1. In samples from batches B, G, I, J and K we found that endotoxin recovered from the spikes exceeded the upper limit. When samples were diluted, the over-response decreased until the endotoxin recovery fell within the accepted range. In addition to endotoxin, the only known molecules able to activate the Limulus cascade are $(1,3)-\beta$-glucan containing structures, which are able to produce false positive results for endotoxin contamination (Cooper et al., 1997). Since it has been previously demonstrated that glucans enhance the LAL response to endotoxins, and that this effect decreases with sample dilution (Roslansky and Novitsky, 1991), we suspected the observed behavior was likely due to $(1,3)-\beta$-glucan contamination.

Therefore, HSA samples were also assayed with an endotoxin-specific LAL reagent (ES-LAL). The enhancing effect was abolished and the final endotoxin concentrations were significantly lower than those obtained by C-LAL, except for batch $\mathrm{H}$ (Tab. 1), thus demonstrating the presence of $(1,3)-\beta$-glucans.

For five batches we found an out of range spike recovery at all dilutions. For that reason, no valid dilution could be stated for the validation/interference assay (Tab. 1). Hence, ES-LAL is not a suitable endotoxin test for HSA.

Interestingly, the spike was recovered at all dilutions in batches G, H and I, which had failed RPT. However, only batches $\mathrm{H}$ and I failed the ES-LAL (Tab. 3).

In general, 1:100 dilutions or higher produced concentrations below the lowest endotoxin concentration of the standard curve (0.01 EU/ml) when using the ES-LAL method. Consequently, the results obtained at 1:10 dilutions are only displayed in the comparative analysis shown in Table 3.

\section{Monocyte activation test (MAT)}

We first determined the linear quantification range under our assay conditions. A linear relationship was found from 0.125 to 1 $\mathrm{EU} / \mathrm{ml}$ for IL-1 $\beta$ and from 0.0625 to $1 \mathrm{EU} / \mathrm{ml}$ for IL-6 (plots not shown), which is in accordance with previous results (Andrade et al., 2003; Poole et al., 2003). The correlation coefficient for both curves was typically greater than 0.99. MVD were 1:32 and 1:64 for IL-1- $\beta$ and IL-6 readouts, respectively.

The interference test for HSA in WB-MAT was conducted three times in clean samples of five different batches. As the quantification range in MAT was up to $1 \mathrm{EU} / \mathrm{ml} \mathrm{LPS}$ with both cytokine readouts, and the spikes were $0.5 \mathrm{EU} / \mathrm{ml}$, it was only possible to quantify an enhancing effect up to $200 \%$. Here we found an enhanced IL-6 response to LPS in the less diluted samples, which was abrogated with sample dilution. This enhanced response was not evidenced with IL-1 $\beta$ (Tab. 2).

The MinVDs for 20\% HSA were 1:32 and 1:4 for IL-6 and IL-1 $\beta$ readout, respectively, and these dilutions were chosen for the assay. Batches $\mathrm{G}$ and $\mathrm{H}$ clearly failed MAT with both 
Tab. 1: Interference test of $20 \%$ HSA in Conventional and Endotoxin-Specific LAL assays

\begin{tabular}{|c|c|c|c|c|c|c|}
\hline \multirow{2}{*}{ Batch } & \multirow{2}{*}{ Dilution } & \multicolumn{2}{|c|}{ C-LAL } & \multirow{2}{*}{ Dilution } & \multicolumn{2}{|c|}{ ES-LAL } \\
\hline & & Concentration $(\mathrm{EU} / \mathrm{ml})^{\mathrm{a}}$ & Spike recovery (\%) & & Concentration $(\mathrm{EU} / \mathrm{ml})^{\mathrm{a}}$ & Spike recovery (\%) \\
\hline \multirow[t]{4}{*}{$A$} & 10 & $23.46 \pm 0.709$ & 52.2 & 10 & $0.05 \pm 0.006$ & 52.4 \\
\hline & 100 & $0.51 \pm 0.021$ & 158.0 & 100 & $<0.01$ & 39.4 \\
\hline & 300 & $0.16 \pm 0.002$ & 170.2 & 400 & $<0.01$ & 41.8 \\
\hline & 500 & $0.12 \pm 0.009$ & 121.4 & & & \\
\hline \multirow[t]{4}{*}{ B } & 10 & $2.33 \pm 0.069$ & 230.6 & 10 & $0.06 \pm 0.003$ & 32.6 \\
\hline & 100 & $0.15 \pm 0.011$ & 75.1 & 100 & $<0.01$ & 37.2 \\
\hline & 300 & $0.09 \pm 0.004$ & 62.7 & 400 & $<0.01$ & 38.4 \\
\hline & 500 & $0.05 \pm 0.001$ & 52.4 & & & \\
\hline \multirow[t]{4}{*}{$\mathrm{D}$} & 10 & $1.27 \pm 0.025$ & 147.7 & 10 & $0.05 \pm 0.002$ & 70.8 \\
\hline & 100 & $0.11 \pm 0.004$ & 132.7 & 100 & $<0.01$ & 41.6 \\
\hline & 300 & $0.06 \pm 0.004$ & 175.1 & 400 & $<0.01$ & 40.8 \\
\hline & 500 & $0.04 \pm 0.003$ & 82.2 & & & \\
\hline \multirow[t]{4}{*}{$\mathrm{F}$} & 10 & $2.33 \pm 0.105$ & 164.1 & 10 & $0.08 \pm 0.006$ & 49.0 \\
\hline & 100 & $0.19 \pm 0.004$ & 103.0 & 100 & $<0.01$ & 39.7 \\
\hline & 300 & $0.08 \pm 0.001$ & 78.0 & 400 & $<0.01$ & 35.3 \\
\hline & 500 & $0.05 \pm 0.004$ & 129.4 & & & \\
\hline \multirow[t]{4}{*}{$\mathrm{G}$} & 10 & $0.63 \pm 0.000$ & 206.9 & 10 & $0.14 \pm 0.000$ & 69.5 \\
\hline & 100 & $0.05 \pm 0.004$ & 107.0 & 100 & $<0.01$ & 50.9 \\
\hline & 300 & $0.03 \pm 0.001$ & 75.9 & 400 & $<0.01$ & 56.9 \\
\hline & 500 & $0.08 \pm 0.003$ & 99.9 & & & \\
\hline \multirow[t]{4}{*}{$\mathrm{H}$} & 10 & $4.62 \pm 0.000$ & 136.8 & 10 & $1.74 \pm 0.064$ & 55.2 \\
\hline & 100 & $0.25 \pm 0.000$ & 99.0 & 100 & $0.13 \pm 0.012$ & 56.8 \\
\hline & 300 & $0.07 \pm 0.004$ & 93.6 & 400 & $<0.01$ & 57.7 \\
\hline & 500 & $0.04 \pm 0.001$ & 82.6 & & & \\
\hline \multirow[t]{4}{*}{ I } & 10 & $3.10 \pm 0.037$ & 529.0 & 10 & $0.41 \pm 0.019$ & 77.6 \\
\hline & 100 & $0.13 \pm 0.001$ & 109.8 & 100 & $0.04 \pm 0.01$ & 53.4 \\
\hline & 300 & $0.03 \pm 0.001$ & 90.2 & 400 & $<0.01$ & 59.8 \\
\hline & 500 & $0.03 \pm 0.001$ & 81.6 & & & \\
\hline \multirow[t]{4}{*}{$\mathrm{J}$} & 10 & $1.95 \pm 0.046$ & 213.0 & 10 & $0.11 \pm 0.008$ & 41.2 \\
\hline & 100 & $0.09 \pm 0.004$ & 81.6 & 100 & $<0.01$ & 40.2 \\
\hline & 300 & $0.04 \pm 0.001$ & 57.5 & 400 & $<0.01$ & 37.1 \\
\hline & 500 & $0.02 \pm 0.001$ & 81.1 & & & \\
\hline \multirow[t]{4}{*}{$\mathrm{K}$} & 10 & $3.21 \pm 0.002$ & 255.0 & 10 & $0.13 \pm 0.004$ & 38.8 \\
\hline & 100 & $0.26 \pm 0.011$ & 189.8 & 100 & $<0.01$ & 44.0 \\
\hline & 300 & $0.09 \pm 0.006$ & 105.0 & 400 & $<0.01$ & 48.3 \\
\hline & 500 & $0.06 \pm 0.004$ & 99.9 & & & \\
\hline \multirow[t]{4}{*}{$\mathrm{P}$} & 10 & $5.30 \pm 0.156$ & 100.8 & 10 & $0.19 \pm 0.006$ & 36.1 \\
\hline & 100 & $0.17 \pm 0.002$ & 81.5 & 100 & $<0.01$ & 36.0 \\
\hline & 300 & $0.06 \pm 0.001$ & 52.3 & 400 & $<0.01$ & 40.1 \\
\hline & 500 & $0.03 \pm 0.001$ & 51.0 & & & \\
\hline
\end{tabular}

a The values are the means of three replicates with S.D. 
Tab. 2: Endotoxin recoveries (\%) obtained in the interference test with $20 \%$ HSA in WB-MAT using IL-1 1 and IL-6 as readout.

\begin{tabular}{|c|c|c|c|c|c|c|c|}
\hline \multirow{2}{*}{ Readout } & \multirow{2}{*}{ Batch } & \multicolumn{5}{|c|}{ Dilutions } & \multirow[b]{2}{*}{$1 / 64$} \\
\hline & & $1 / 2$ & $1 / 4$ & $1 / 8$ & $1 / 16$ & $1 / 32$ & \\
\hline \multirow[t]{5}{*}{ IL-1 $\beta$} & A & 169.8 & 94.3 & 133.4 & 106.4 & 80.5 & 76.0 \\
\hline & B & 117.8 & 160.7 & 158.4 & 90.9 & 83.8 & 92.0 \\
\hline & C & 12.2 & 145.7 & 149.7 & 120.9 & 76.6 & 58.0 \\
\hline & D & 70.0 & 72.2 & 98.9 & 148.1 & 132.6 & 109.9 \\
\hline & $F$ & 66.8 & 101.2 & 96.2 & 78.9 & 69.4 & 75.0 \\
\hline \multirow[t]{5}{*}{ IL-6 } & A & N.D. & N.D. & $>200$ & $>200$ & 67.7 & 112.7 \\
\hline & B & N.D. & N.D. & $>200$ & $>200$ & 129.0 & 125.5 \\
\hline & C & N.D. & N.D. & $>200$ & 97.4 & 38.6 & 154.0 \\
\hline & D & N.D. & N.D. & $>200$ & $>200$ & $>200$ & 185.1 \\
\hline & $\mathrm{F}$ & N.D. & N.D. & $>200$ & $>200$ & 157.9 & 133.3 \\
\hline
\end{tabular}

Recovery was calculated using the mean value of endotoxin equivalent concentrations $(\mathrm{EEU} / \mathrm{ml})$ of the spiked $\mathrm{HSA}$ at $0.5 \mathrm{EU} / \mathrm{ml}$ (S) and the un-spiked samples (US) as follows: \% Recovery $=((\mathrm{S}-\mathrm{US}) / 0.5) \times 100$.

In samples labeled ND (not determined), it was not possible to quantify the endotoxin recovery since both the spike and unspiked dilutions produced saturating spectrophotometric signals.

Tab. 3: Comparative results of pyrogen evaluation of HSA by Monocyte Activation Test, Rabbit Pyrogen Test and Endotoxin-Specific-LAL

\begin{tabular}{|c|c|c|c|c|}
\hline Batch & $\operatorname{MAT}(\mathrm{IL}-1 \beta)(\mathrm{EEU} / \mathrm{ml})^{\mathrm{a}}$ & MAT (IL-6) (EEU/ml) ${ }^{\mathrm{a}}$ & $\operatorname{RPT}(\Sigma \Delta \mathrm{T})\left({ }^{\circ} \mathrm{C}\right)$ & ES-LAL $(E U / m l)^{a}$ \\
\hline A & $0.81 \pm 0.242^{P}$ & $<2^{P}$ & $0.60^{P}$ & $0.52 \pm 0.003^{P}$ \\
\hline B & $0.93 \pm 0.224^{P}$ & $2.22 \pm 0.921^{P}$ & $0.05^{P}$ & $0.61 \pm 0.029^{P}$ \\
\hline C & $1.29 \pm 0.245^{P}$ & $5.10 \pm 0.183^{F}$ & $0.10^{P}$ & $1.30 \pm 0.038^{P}$ \\
\hline $\mathrm{D}$ & $1.29 \pm 0.406^{P}$ & $2.52 \pm 0.760^{P}$ & $0.25^{P}$ & $0.54 \pm 0.017^{P}$ \\
\hline$E$ & $1.15 \pm 0.364^{P}$ & $2.83 \pm 0.609^{P}$ & $0.35^{P}$ & $0.64 \pm 0.077^{P}$ \\
\hline $\mathrm{F}$ & $1.07 \pm 0.517^{P}$ & $2.62 \pm 1.084^{P}$ & $0.35^{P}$ & $0.82 \pm 0.062^{P}$ \\
\hline$G$ & $>8^{\mathrm{F}}$ & $8.64 \pm 1.28^{F}$ & 5.30 (first retest) $^{\mathrm{F}}$ & $1.39 \pm 0.000^{P}$ \\
\hline $\mathrm{H}$ & $>8^{\mathrm{F}}$ & $14.67 \pm 4.31^{\mathrm{F}}$ & 5.70 (first retest) $^{\mathrm{F}}$ & $15.35 \pm 2.480^{F}$ \\
\hline I & $3.57 \pm 0.795^{P}$ & $3.85 \pm 0.75^{P}$ & 7.05 (third retest) $^{\mathrm{F}}$ & $4.07 \pm 0.187^{F}$ \\
\hline$J$ & $<0.5^{\mathrm{P}}$ & $<2^{P}$ & $0.55^{P}$ & $1.09 \pm 0.077^{P}$ \\
\hline $\mathrm{K}$ & $<0.5^{P}$ & $<2^{P}$ & $0.50^{P}$ & $1.33 \pm 0.039^{P}$ \\
\hline L & $<0.5^{P}$ & $2.35 \pm 0.022^{P}$ & 1.85 (first retest) $^{\mathrm{P}}$ & $1.03 \pm 0.025^{P}$ \\
\hline$M$ & $1.04 \pm 0.157^{P}$ & $<2^{P}$ & $0.55^{P}$ & $0.96 \pm 0.035^{P}$ \\
\hline $\mathrm{N}$ & $0.68 \pm 0.182^{P}$ & $2.36 \pm 1.006^{P}$ & $0.20^{P}$ & $0.72 \pm 0.000^{P}$ \\
\hline $\mathrm{O}$ & $0.80 \pm 0.233^{P}$ & $2.72 \pm 0.985^{P}$ & $0.15^{\mathrm{P}}$ & $1.43 \pm 0.048^{P}$ \\
\hline$P$ & $1.48 \pm 0.280^{P}$ & $<2^{P}$ & $0.35^{P}$ & $1.93 \pm 0.063^{P}$ \\
\hline
\end{tabular}

${ }^{a}$ The values are the means of three replicates with S.D.

$P$ Passed the assay

F Failed the assay

cytokine readouts and this was in accordance with the RPT results, where these batches failed in the first retest. Batch I failed RPT in the third retest and contained an endotoxin equivalent concentration around the $4 \mathrm{EEU} / \mathrm{ml}$ limit as determined with WB-MAT with both cytokine readouts (Tab. 3).

There were noticeably higher endotoxin equivalent concentrations obtained with the IL- 6 readout than with IL- $1 \beta$, insomuch that batch $\mathrm{C}$ would fail the test with the IL- 6 readout, whereas it passed MAT/IL-1 $\beta$ and RPT (Tab. 3).
Cytokine concentration

IL-1 $\beta$ is an effective stimulator of IL-6 gene expression and protein translation (Dinarello, 2004), whereas IL-6 has an inhibitory effect on IL-1 $\beta$ production (Fenton, 1992; Watkins et al., 1999). As IL-1 $\beta$ and/or IL-6 are conceivable contaminants of blood-derived products, the concentrations of both cytokines were determined in undiluted HSA from batch $\mathrm{C}$.

A linear relationship between IL-1 $\beta$ and IL-6 vs. OD was typically found between 7-500 pg/ml and 1-200 pg/ml, respective- 


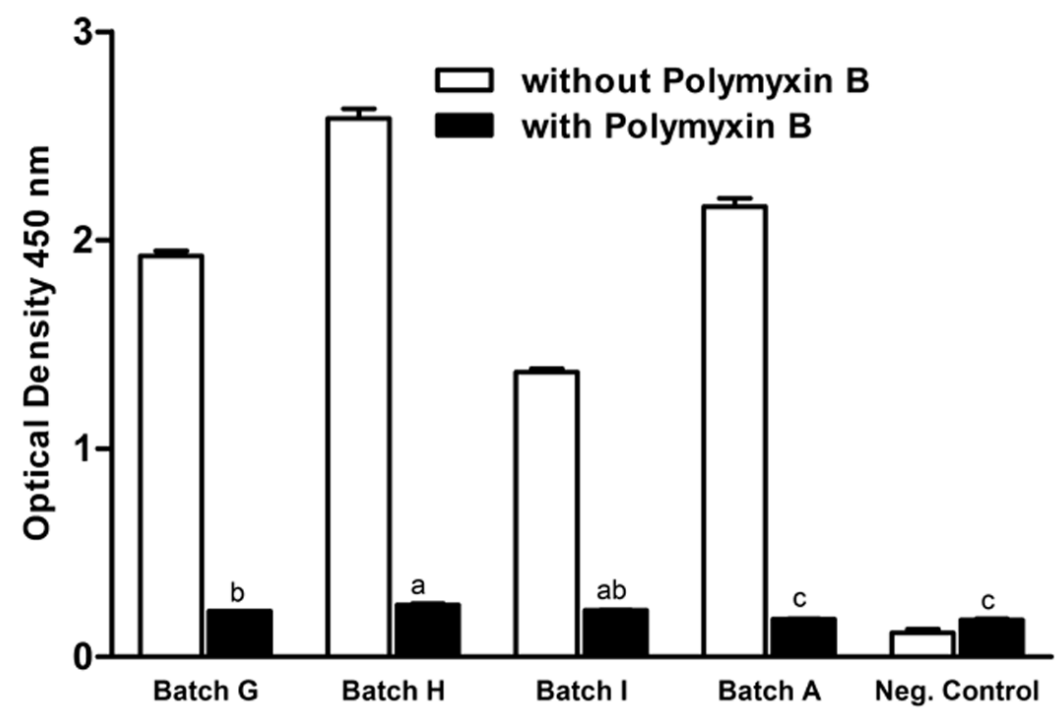

Fig. 1: Effect of polymyxin B on MAT/IL-1 $\beta$ response in pyrogenic HSA

Each sample was incubated with whole blood with and without polymyxin $B$, and the IL-1 $\beta$ response in the supernatant was determined by ELISA. Batch A: Clean HSA sample spiked with $10 \mathrm{EU} / \mathrm{ml}$ (assay control); Batches G, H and I failed the Rabbit Pyrogen Test. Negative control: pyrogen-free physiological saline. All HSA samples were assayed at 1/4 dilution. Data are represented as mean \pm SD of two separate experiments. Groups with different letters were significantly different at $p<0.05$

1y. Furthermore, OD values above 2 units were normally out of the linear phase of the dose response curve for both cytokines' standard curves under our assay conditions (plots not shown). IL-6 was not detected (less than $1 \mathrm{pg} / \mathrm{ml}$ ), and IL-1 $\beta$ was $43 \mathrm{pg}$ / $\mathrm{ml}$. Considering samples were diluted at least 32 times to overcome interferences in the MAT/IL-6 assay and the additional sample dilution in the assay system, we could assume that differences in estimated endotoxin concentration between both readouts were due neither to the induction of IL- 6 secretion by IL- $1 \beta$, nor by inhibition of IL- $1 \beta$ production by IL- 6 .

\section{Differentiation between endotoxin and non-endotoxin pyrogen contamination}

In this experiment we attempted to define the contaminating source in the three batches of HSA that were pyrogenic as determined by RPT. Pool et al. (1999) demonstrated that using Polymyxin B (PMB) combined with MAT allows differentiation between endotoxin and non-endotoxin pyrogenic contamination of pharmaceuticals. PMB efficiently inhibits the cytokine response to LPS, including LPS from PMB-resistant strains of bacteria, by promoting a change in the aggregate state of LPS into an inactive LPS micelle (Gutsmann et al., 2010).

We first evaluated the effect of PMB in our assay conditions in a clean batch of HSA spiked with $10 \mathrm{EU} / \mathrm{ml}$ (Batch A in Fig. $1)$. There were no significant differences $(p<0.05)$ in cytokine production between endotoxin-spiked HSA treated with PMB and the assay control; thus demonstrating that endotoxin activity was completely abrogated. By using the same approach, PMB reduced between 83 and $90 \%$ of the cytokine response in the contaminated batches (Fig. 1), indicating that they were largely contaminated with endotoxin. Nevertheless, a low cytokine re- sponse significantly different $(\mathrm{p}<0.05)$ to the assay control remained in the presence of PMB. This appears to be derived from the presence of non-endotoxin pro-inflammatory entities.

\section{Discussion}

Testing of HSA for pyrogens has been included in several studies to benchmark the replacement of the rabbit pyrogen test with the LAL (Bleeker et al., 1985; Hegedüs and Benkö, 1977; Hochstein et al., 1979; Watt et al., 1979) and the MAT (Poole et al., 1988a,b; Taktak et al., 1991). The LAL has proven to be a very sensitive and effective assay to detect endotoxin from Gram negative bacteria. It has been employed with success for more than 20 years as an end-product endotoxin test in parenteral pharmaceuticals. However, pyrogenicity testing of HSA is still regulated mainly by the RPT, despite some drawbacks of this assay with regard to testing this product (Hartung et al., 2001). In the present study, sixteen batches of $20 \%$ HSA were assayed by LAL, RPT, and WB-MAT determining the IL- $1 \beta$ and IL- 6 response in order to provide parallel testing data to support the safety and reliability of MAT against the established LAL and RPT.

It was found that all HSA batches were contaminated with $(1,3)-\beta$-glucans as shown by the differences found between conventional and endotoxin-specific LAL assay. The main source of contaminating $(1,3)$ - $\beta$-glucans in blood-derived products are cellulose membranes commonly used for clarification (Buchacher et al., 2010; Nagasawa et al., 2003; Ochiai et al., 2010; Ohata et al., 2003; Usami et al., 2002).

Previous studies have indicated that the endotoxin concentration in HSA preparations determined with the ES-LAL reagent 
correlates with the RPT result, and as the endotoxin spikes were recovered within the accepted range, the authors concluded that ES-LAL could be suitable for testing HSA for endotoxins (Asakawa et al., 1994; Fujiwara et al., 1990). The same has been described for other blood-derived products (Naito et al., 1992, Ochiai et al., 2010). The LAL assay is implemented in Japan for pyrogen testing of HSA (Ministery of Health and Welfare, Japanese government, 2006).

However, we encountered problems with endotoxin recovery in the interference test with both C-LAL and ES-LAL that invalidate the use of LAL as an end-product endotoxin test. Failure to recover endotoxin from HSA with LAL has been documented previously (Hochstein et al., 1979). Those authors suggested that HSA masks certain amounts of endotoxin until saturation occurs and therefore the LAL test is not able to fully detect the endotoxin spikes (Hochstein et al., 1979). The masking effect of LPS by HSA occurs by non-electrostatic interaction between both molecules, hindering the 4'-phosphate group essential for LAL reactivity inside the aggregate (Jürgens et al., 2002). This could explain why the only batches in which the spike was recovered in the interference test with ES-LAL were those that were pyrogenic in the RPT.

On the other hand, we have found an enhanced response of monocytes to LPS in the MAT/IL-6 interference test, which is probably due to the presence of cellulose-derived $(1,3)$ - $\beta$-glucans. Similar findings have been described earlier (Kikkert et al., 2007). Conversely, others have found that glucans from C. albicans suppress the LPS-induced IL-6 response (Nakagawa et al., $2003)$. The biological activity of $(1,3)-\beta$-glucans in vertebrates is complex and relates to their origin, size, molecular structure, purity, and cell type or receptor(s) involved (for reviews see Brown, 2006; Goodridge et al., 2009; Reid et al., 2009; Tsoni and Brown, 2008).

In the current study, it also was found that endotoxin equivalent concentrations obtained with MAT/IL-6 were usually higher than with MAT/IL- $1 \beta$, which could be due to $(1,3)-\beta$-glucans having directly elicited an IL-6 response in monocytes. Our thinking is based on previous reports stating that glucans of intermediate or low molecular weights could induce an IL-6 response but not an IL-1 $\beta$ response (Brown and Gordon, 2003, 2005). We have not found information on the molecular weight of $(1,3)-\beta$-glucans extracted from cellulose filters. However, they might be similar to those extracted from cellulose membrane components of hollow-fiber hemodialyzers, which have a molecular weight of around 24,000 (Pearson et al., 1984, 1987). This issue is also contradictory in the literature, because other reports indicate that glucans from cellulose filters (Kikkert et al., 2007) or from yeast (Nakagawa et al., 2002) do not induce IL-6 release in monocytes or monocytoid cell lines. This issue would require further studies.

Here we have found that, in a similar fashion to the conventional LAL, glucan contamination can provoke two different results in MAT/IL-6: 1) false positive for endotoxin, and 2) enhanced response to endotoxin.

The direct IL-6 response to glucan contamination by monocytes in MAT/IL-6, along with its higher sensitivity, were responsible for the saturating response in less diluted, un-spiked samples observed in MAT/IL-6 interference test (Tab. 2). As IL6 is a well-known endogenous pyrogen, it would be conceivable that such burst release could bring about a pyrogenic episode. Yet, many of the HSA samples were contaminated with glucans and were non-pyrogenic in rabbits, probably because much higher levels of IL- 6 than IL- $1 \beta$ are required to produce fever in humans and in rabbits (Dinarello, 2004).

It has already been stated that $(1,3)-\beta$-glucans are not pyrogenic (Roslansky and Novitsky, 1991), including those extracted from cellulose material (Buchacher et al., 2010; Pearson et al., 1984). Consequently, WB-MAT using the IL- $1 \beta$ readout seems to resemble the pyrogenic response in rabbits better than IL-6.

Even though clinical data demonstrating a pyrogenic effect of $(1,3)-\beta$-glucans in humans is still lacking, we should be aware that these are biologically active molecules which, like endotoxins, are classified as pathogen-associated molecular patterns (PAMP) (Medzhitov and Janeway, 2000). Therefore, as suggested by others (Kikkert et al., 2007), their presence as contaminants in pharmaceuticals could be undesirable. Further, their synergistic effect on the IL-6 response to LPS by monocytes, even at sub-threshold pyrogenic response levels of LPS, could imply a serious deleterious effect if this combination is administered to patients, which may not necessarily be revealed by a fever reaction. Currently, however, there is no explicit regulation concerning the presence of this contaminant in pharmaceuticals.

Earlier reports described the LAL assay as failing to detect pyrogenic HSA probably contaminated with non-endotoxin pyrogens as assessed by MAT (Pool et al., 1999; Taktak et al., 1991). However, the three pyrogenic batches identified by RPT in the current study were contaminated with endotoxin and one of them passed the LAL assay, whereas all three failed MAT using IL- $1 \beta$ or IL- 6 as readout, demonstrating a good correlation between MAT and RPT for production-related pyrogenic and non-pyrogenic HSA samples.

Dehus et al. have demonstrated that the WB-MAT response to endotoxins does not always correlate with LAL assay results (Dehus et al., 2006), probably because the basic requirements for the activation of Limulus hemocytes and the induction of cytokines by endotoxins in human monocytes are quite different (Brandenburg et al., 2009; Gutsmann et al., 2010).

In the current study we have shown that the WB-MAT is a suitable and reliable assay for pyrogenicity testing in HSA, which overcomes essential disadvantages of the other pyrogen tests for this product. WB-MAT is not influenced by an interaction between HSA and LPS (Brandenburg et al., 2009), and as it closely resembles the human physiological response (Hartung, 2001), it should best describe the potential activity of endotoxins in HSA formulations, providing higher safety levels. Our results, together with previous studies, support the replacement of RPT with MAT as final release test for pyrogens in HSA, and probably also in other blood-derived products and therapeutic proteins.

\section{References}

Andrade, S., Silveira, R., Schmidt, C., et al. (2003). Comparative evaluation of the human whole blood and human peripheral blood monocyte tests for pyrogens. Int. J. Pharm. 265, 115-124. 
Asakawa, S., Fujiwara, H., Naito, S., et al. (1994). Application of the limulus test for practical quality control on endotoxin content in commmercial human serum albumin (HSA) products. In comparison with the rabbit pyrogen test. J. Pharm. Soc. Jpn. 114, 888-893.

Bleeker, W. K., Kannegieter, E. M., Bakker, J. C., and Loos, J. A. (1985). Endotoxin in blood products: correlation between the Limulus assay and the rabbit pyrogen test. Prog. Clin. Biol. Res. 189, 293-303.

Brandenburg, K., Howe, J., Gutsman, T., and Garidel, P. (2009). The expression of endotoxic activity in the Limulus test as compared to cytokine production in immune cells. Curr. Med. Chem. 16, 2653-2660.

Brown, G. D., and Gordon, S. (2003). Fungal $\beta$-Glucans and mammalian immunity. Immunity 19, 311-315.

Brown, G. D., and Gordon, S. (2005). Immune recognition of fungal beta-glucans. Cell Microbiol. 7, 471-479.

Brown, G. D. (2006). Dectin-1: a signalling non-TLR patternrecognition receptor. Nat. Rev. Immunol. 6, 33-43.

Buchacher, A., Krause, D., Wiry, G., and Weinberger, J. (2010). Elevated endotoxin levels in human intravenous immunoglobulin concentrates caused by $(1 \rightarrow 3)$-beta-D-Glucans. PDA J. Pharm. Sci. Technol. 64, 536-544.

Cooper, J. F., Weary, M. E., and Jordan, F. T. (1997). The impact of non-endotoxin LAL-reactive materials on Limulus amebocyte lysate analyses. PDA J. Pharm. Sci. Technol. 51, 2-6.

Daneshian, M., von Aulock, S., and Hartung, T. (2009). Assessment of pyrogenic contaminations with validated human whole-blood assay. Nat. Protoc. 14, 1709-1721.

Dehus, O., Hartung, T., and Hermann, C. (2006). Endotoxin evaluation of eleven lipopolysaccharides by whole blood assay does not always correlate with Limulus amebocyte lysate assay. J. Endotoxin Res. 12, 171-180.

Dinarello, C. (2004). Infection, fever, and exogenous and endogenous pyrogens: some concepts have changed. J. Endotoxin Res. 10, 201-222.

EMEA (2009). Guideline of the replacement of rabbit pyrogen testing by an alternative test for plasma derived medicinal products. London, U.K.: European Medicines Agency. http:// www.emea europa.edu/docs/en_GB/document_library/Scientific_guideline/2009/09/WC500003599.pdf

ESAC Statement (2006). http://ecvam.jrc.it/ft_doc/ESACstatementpyrogenicity20060321.pdf

EP - European Pharmacopoeia (2007). 6.0: 2.6.8. Pyrogens.

EP - European Pharmacopoeia (2010). 6.7: 2.6.30. Monocyte Activation Test.

FDA (1987). Guideline on validation of the limulus amebocyte lysate test as an end-product endotoxin test for human and animal parenteral drugs, biological products, and medical devices. Washington DC, U.S. Food and Drug Administration. http://www.fda.gov/downloads/BiologicsBloodVaccines/ GuidanceComplianceRegulatoryInformation/Guidance/ Blood/UCM080966.pdf

FDA (2009). Letter from Norris E. Alderson, Ph.D., FDA Associate Commissioner for Science, to RADM William S. Stokes, Director NICEATM. http://iccvam.niehs.nih.gov/ methods/pyrogen/transmitNov08/FDA-Response.pdf
Fenton, M. J. (1992). Transciptional and porst-transcriptional regulation of interleukin 1 gene expression. Int. J. Immunopharmacol. 14, 401-411.

FNM (2006). Formulario Nacional de Medicamentos, Ministerio de Salud Pública, Ecimed, Cuba. http://www.bvs.sld.cu/libros/formulario_nacional_medicamentos/11_productos_sanguineos.pdf

Fujiwara, H., Ishida, S., Shimazaki, Y., et al. (1990). Measurement of endotoxin in blood products using an endotoxin-specific Limulus test reagent and its relation to pyrogenic activities in rabbit. J. Pharm. Soc. Jpn. 110, 332-340.

Gaines-Das, R. E., and Poole, S. (1993). The international standard for interleukin-6: Evaluation in an international collaborative study. J. Immunol. Methods. 160, 147-153.

Goodridge, H. S., Wolf, A. J., and Underhill, D. M. (2009). Beta-glucan recognition by the innate immune system. Immunol. Rev. 230, 38-50.

Gutsmann, T., Howe, J., Zähringer, U., et al. (2010). Structural prerequisites for endotoxic activity in the Limulus test as compared to cytokine production in mononuclear cells. Innate Immun. 16, 39-47.

Hartung, T., Aaberge, I., Berthold, S., et al. (2001). Novel pyrogen tests based on the human fever reaction. The report and recommendations of ECVAM Workshop 43. European Centre for the Validation of Alternative Methods. ATLA 29, 99-123.

Hegedüs, L., and Benkö, A. (1977). Comparative pyrogen reactivity of rabbit and man to human albumin and immunoglobulin solutions. Dev. Biol. Stand. 34, 127-133.

Hochstein, H. D., Seligmann Jr, E. B., Marquina, R. E., and Rivera, E. (1979). Limulus amebocyte lysate testing of nomral serum albumin (human) in the United States since 1975. Dev. Biol. Stand. 44, 35-42.

Hoffmann, S., Peterbauer, A., Schindler, S., et al. (2005). International validation of novel pyrogen tests based on human monocytoid cells. J. Immunol. Methods 298, 161-173.

Huszar, G., Jenei, B., Szabo, G., and Medgyesi, G. (2002). Detection of pyrogens in intravenous IgG preparations. Biologicals 30, 77-83.

ICCVAM (2008). ICCVAM test method evaluation report: validation status of five in vitro test methods proposed for assessing potential pyrogenicity of pharmaceuticals and other products. NIH Publication Number 08-6392. Research triangle Park, NC, National Institute of environmental Health Sciences. http://iccvam.niehs.nih.gov/methods/pyrogen/pyr_tmer.htm

ICCVAM (2009). In vitro pyrogen test methods. http://iccvam. niehs.nih.gov/methods/pyrogen/pyrogen.htm

Jürgens, G., Müller, M., Garidel, P., et al. (2002). Investigation into the interaction of recombinant human serum albumin with Relipopolysaccharide and lipid A. J. Endotoxin Res. 8, 115-126.

Kikkert, R., Bulder, I., de Groot, E., et al. (2007). Potentiation of Toll-like receptor-induced cytokine production by $(1 \rightarrow 3)-\beta$-D-glucans: implications for the monocyte activation test. J. Endotoxin Res. 13, 140-149.

Mc Clelland, B. (1990). Human albumin solutions. In M. Contreras (ed.), ABC of transfusion (35-37). London: BMJ Press.

Medzhitov, R., and Janeway, C. A. (2000). Innate Immunity. $N$. Engl. J. Med. 343, 338-344. 
Ministry of Health and Welfare, Japanese Government (2006). Minimun requirements for biological products.

Montag, T., Spreitzer, I., Loschner, B., et al. (2007). Safety testing of cell-based medicinal products: opportunities for the monocyte activation test for pyrogens. ALTEX 24, 81-89.

Nagasawa, K., Yano, T., Kitabayashi, G., et al. (2003). Experimental proof of contamination of blood components by $(1 \rightarrow 3)$-beta-D-glucan caused by filtration with cellulose filters in the manufacturing process. J. Artif. Organs 6, 49-54.

Naito, S., Fijuwara, H., Asakawa, S., et al. (1992). Application of the limulus amebocyte test to measurment of endotoxin in therapeutic human plasma protein fraction. Comparison with the rabbit pyrogen test. J. Pharm. Soc. Jpn. 112, 551-556.

Nakagawa, Y., Maeda, H. and Murai, T. (2002). Evaluation of the in vitro pyrogen test system based on proinflammatory cytokine release from human monocytes: comparison with a human whole blood culture test system and with the rabbit pyrogen test. Clin. Diagn. Lab. Immunol. 9, 588-597.

Nakagawa, Y., Ohno, N., and Murai, T. (2003). Suppression by Candida albicans $\beta$-Glucan of cytokine release from activated human monocytes and from $\mathrm{T}$ cells in the presence of monocytes. J. Infect. Dis. 187, 710-713.

Ochiai, M., Yamamoto, A., Naito, S., et al. (2010). Applicability of bacterial endotoxins test to various blood products by the use of endotoxin-specific lysates. Biologicals 38, 629-636.

Ohata, A., Usami, M., Horiuchi, T., et al. (2003). Release of $(1,3)$-beta-D-glucan from depth-type membrane filters and their in vitro effects on proinflammatory cytokine production. Artif. Organs 27, 728-735.

Pearson, F. C., Bohon, J., Lee, W., et al. (1984). Characterization of Limulus amoebocyte lysate-reactive material from hollowfiber dialyzers. Appl. Environ. Microbiol. 48, 1189-1196.

Pearson, F. C., Caruana, R., Burkart, J., et al. (1987). The use of the Limulus amebocyte lysate assay to monitor hemodialyzer-associated soluble cellulosic material (LAL-reactive material). Prog. Clin. Biol. Res. 231, 211-222.

Pool, M., and McLeod, B. (1995). Pyrogen reactions to human serum albumin during plasma exchange. J. Clin. Apher. 10, 81-84.

Pool, E., Johaar, G., James, S., et al. (1999). Differentiation between endotoxin and non-endotoxin pyrogens in human albumin solutions using an ex vivo whole blood culture assay. $J$. Immunoassay 20, 79-89.

Poole, S., Thorpe, R., Meager, A., and Gearing, A. (1988a). Assay of pyrogenic contamination in pharmaceuticals by cytokine release from monocytes. Dev. Biol. Stand. 69, 121-123.

Poole, S., Thorpe, R., Meager, A., et al. (1988b). Detection of pyrogens by cytokine release. Lancet 16, 130 .

Poole, S., and Gaines-Das, R. E. (1991). The international standards for interleukin-1alpha and interleukin-1beta: Evaluation in an international collaborative study. J. Immunol. Methods 142, 1-13.

Poole, S., Dawson, P., and Gaines-Das, R. E. (1997). Second international standard for endotoxin: calibration in an international collaborative study. J. Endotoxin Res. 4, 221-231.

Poole, S., Mistry, Y., Ball, C., et al. (2003). A rapid 'one-plate' in vitro test for pyrogens. J. Immunol. Methods 274, 209-220.
Reid, D. M., Gow, N. A., and Brown, G. D. (2009). Pattern recognition: recent insights from Dectin-1. Curr. Opin. Immunol. 21, 30-37.

Roslansky, P. F., and Novitsky, T. J. (1991). Sensitivity of Limulus amebocyte lysate (LAL) to LAL-reactive glucans. J. Clin. Microbiol. 99, 2477-2483.

Spreitzer, I., Fischer, M., Hartzsch, K. et al. (2002). Comparative study of rabbit pyrogen test and human whole blood assay on human serum albumin. ALTEX 19, 73-75.

Steere, A. C., Rifaat, M. K., Seligmann Jr, E. B., et al. (1978). Pyrogens reactions associated with the infusion of normal serum albumin (human). Transfusion 18, 102-107.

Taktak, Y., Selkirk, S., Bristow, A., et al. (1991). Assay of pyrogens by interleukin-6 release from monocytic cell lines. J. Pharm. Pharmacol.43, 578-582.

Tsoni, S., and Brown, G. (2008). Beta-Glucans and dectin-1. Ann. N.Y. Acad. Sci. 1143, 45-60.

Usami, M., Ohata, A., Horiuchi, T., et al. (2002). Positive $(1 \rightarrow 3)$-beta-D-glucan in blood components and release of $(1 \rightarrow 3)$-beta-D-glucan from depth-type membrane filters for blood processing. Transfusion 42, 1189-1195.

Watkins, L. R., Nguyen, K. T., Lee, J. E., and Maier. S. F. (1999). Dynamic regulation of proinflammatory cytokines. Adv. Exp. Med. Biol. 461, 153-178.

Watt, J., Grant, W., and Patterson, M. (1979). Experience of changing pyrogenicity in albumin solutions. Dev. Biol. Stand. 44, 31-34.

Yokota, H., Kiyonaga, H., Kaniwa, H., and Shibanuma, T. (2001). Adsorption of endotoxins on glass in the presence of cationic proteins. J. Pharm. Biomed. Anal. 25, 1001-1007.

\section{Acknowledgements}

The authors would like to express their gratitude to Bettina Loeschner from the Paul Ehrlich Institute, Germany, for her unconditional support, and to Mary Morgan from Dr. Spock Corporation for English corrections.

\section{Correspondence to}

Rolando Perdomo-Morales

Center for Pharmaceuticals Research and Development

Ave. 26 No. 1605. Plaza. CP 10400

Havana, Cuba

e-mail: rolando.perdomo@infomed.sld.cu 